\title{
Diacronie
}

Studi di Storia Contemporanea

$N^{\circ} 22,2$ | 2015

Costruire

\section{Narrare la storia: la lezione di Jerzy Topolski}

\section{Patrizia Fazzi}

\section{(2) OpenEdition}

\section{Journals}

\section{Edizione digitale}

URL: http://journals.openedition.org/diacronie/2082

DOI: 10.4000/diacronie.2082

ISSN: 2038-0925

\section{Editore}

Association culturelle Diacronie

\section{Notizia bibliografica digitale}

Patrizia Fazzi, « Narrare la storia: la lezione di Jerzy Topolski », Diacronie [Online], № 22, 2 | 2015

documento 11, online dal 01 juin 2015, consultato il 09 octobre 2020. URL : http://

journals.openedition.org/diacronie/2082 ; DOI : https://doi.org/10.4000/diacronie.2082 


\title{
Diacronie
}

N. 22 | 2|2015 Costruire. Rappresentazioni, relazioni, comunità

\section{1/}

\section{Narrare la storia: la lezione di Jerzy Topolski}

\author{
Patrizia FAZZI *
}

L'articolo analizza i principi di metodologia proposti dallo storico polacco Jerzy Topolski in relazione all'evoluzione del pensiero storiografico contemporaneo. L'autore fornisce un'analisi accurata del racconto storico, delle sue strutture retoriche, dell'uso delle fonti e dei condizionamenti ideologici, valoriali e culturali che intervengono nella ricerca e nella stesura del testo. Ne deriva una Storia come scienza non sempre esatta, ma che si avvicina alla verità, e si delinea la figura dello storico che cerca il "contatto" con la realtà passata, nella cui fisionomia entrano il rigore scientifico, la ricerca del metodo, le competenze tecniche, ma anche l'immaginazione e la passione per la conoscenza.

\section{Premessa}

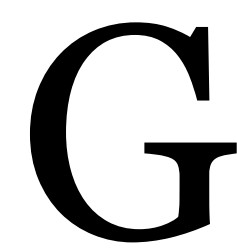
li storici della contemporaneità si trovano oggi costretti a intervenire in un contesto complesso, in cui dilaga in modo invasivo l'uso pubblico della storia e insidiose sono le sfide epistemologiche che investono lo statuto scientifico della disciplina. La prospettiva di soccombere nel confronto con altri mezzi di comunicazione, più efficaci nel trasmettere la conoscenza storica, gli stereotipi espressi dalle vulgate, il rifiuto della complessità dell'argomentazione in favore di percorsi semplificati, provocano disagio e inquietudine. E poi, la dimensione immateriale della rete e il suo utilizzo improprio tendono a far precipitare i capisaldi della tradizione critica delle fonti, oltre a opacizzarne il contesto di origine e di riferimento.

In questo scenario, la lezione di Jerzy Topolski è da cogliere come il tentativo di sistematizzazione del lavoro dello storico, una costruttiva mediazione tra due poli 
opposti: dallo scientismo positivista, ancorato ai suoi modelli nomologici, che assegna all'attività storiografica un posto fra le scienze esatte, al decostruzionismo, che riduce il testo a pura costruzione linguistica, in un percorso di ricerca privo della nozione di "verità" e, dunque, senza rispondenza alcuna con la realtà.

\section{Contestualizzazione storiografica}

Nella pratica storiografica, analitica e di stampo positivista, sono state seguite alcune ipotesi fondamentali che hanno condizionato il lavoro di ricerca: una premessa ontologica, nella convinzione di interpretare il passato come categoria indipendente dal soggetto che lo studia; un ben definito indirizzo epistemologico alla scoperta della verità storica come fine ultimo della ricerca stessa; infine, un orientamento prammatico, nell'intento di ricostruire la verità attraverso la fonte storica ${ }^{1}$. Il realismo degli storici esiste, dunque, anche in virtù del contatto empirico che essi hanno con il passato attraverso le fonti. Del nuovo interesse scientifico sono sintomo le messe a punto di strumenti tecnici, la fondazione di riviste specialistiche e di società di studi storici, all'interno di un clima culturale che ha favorito una trattatistica specificamente metodologica.

A partire dagli anni sessanta del secolo scorso si sono tuttavia incrinate le certezze di stampo positivista e si è cominciato a registrare un crescente interesse per la problematica del racconto storico, in concomitanza con una particolare attenzione al medium linguistico, senza abbandonare il classico metodo scientifico della ricerca. Pur sottolineando la funzione della spiegazione deduttiva come fattore fondamentale per la costruzione strutturale del racconto, si è lasciato spazio a concezioni che non fossero solo ancorate a modelli logici e informativi, aprendo così la strada a nuovi approcci metodologici.

Un nutrito gruppo di studiosi si è chiesto se la spiegazione storica fosse affine o meno a quella in uso nelle scienze esatte e il racconto stesso ha assunto via via il grado di categoria da rivalutare nell'ambito della metodologia della storia, seguendo non solo una ricerca di regole e modelli generalizzanti, ma anche di procedimenti narrativi, retorici e culturali.

La prima opera significativa risale ad Arthur Danto, il quale, senza abbandonare l'indirizzo positivista, ha iniziato a svincolarsi dai modelli logici e dalle proposizioni

\footnotetext{
${ }^{1}$ Tra i più significatori autori si segnalano: Carl Georg Hempel, Maurice Mandelbaum e Patrick Gardiner.
} 
individuali, pur considerando la spiegazione deduttiva un fattore fondamentale per assicurare la coerenza ${ }^{2}$.

È ritornato così al centro della conoscenza storica il dualismo spiegazionecomprensione, con una nuova attenzione nei confronti di quest'ultima. Sotto l'influenza di Robin G. Collingwood e Louis O. Mink, il racconto stesso ha assunto il grado di categoria principale della metodologia della storia, con espliciti richiami alla filosofia di Johann Gustav Droysen, Wilhelm Dilthey e Benedetto Croce. La comprensione delle azioni umane, diventata l'oggetto principale della ricerca, non poteva tuttavia essere disgiunta dalla conoscenza delle categorie di dinamica e struttura del processo storico ${ }^{3}$.

Tra gli autori francesi che hanno scelto la strada del racconto in una prospettiva non positivista, è doveroso citare Michel Foucault, Paul Ricoeur e Paul Veyne4. In particolare quest'ultimo, tra i primi a proporre una vera e propria metodologia a carattere narratologico, si è soffermato a lungo sul concetto di narrazione come "struttura del testo", piuttosto che racconto lineare, sottolineando anche che la storia quantitativa e la storia sociale sono costruite sull'intrigo, essendo quest'ultimo "una mescolanza" molto umana e poco "scientifica" di cause materiali, di fini e di casis. La nozione basilare della narratività non ha nulla a che fare, dunque, con la cronologia, ma rimanda all'atto della sintesi dell'eterogeneo.

Ne deriva una particolare concezione del lavoro dello storico, che lega il suo racconto allo sviluppo dell'intreccio: una costruzione in stretta somiglianza strutturale con il romanzo. Se il tempo "umano" è il tempo "raccontato", il racconto come tale è misura dello stesso farsi "umano" del tempo.

\footnotetext{
${ }^{2}$ A. Danto ha avviato una riflessione sulla ricerca storica, differenziandola dalla ricerca scientifica in quanto "narrazione semplice" e non "spiegazione" dei fatti, che conserva uno statuto meramente condizionale. L'analisi proposizionale non si riduce a mero esercizio di logica astratta, ma alla penetrazione delle strutture costitutive della realtà. Con questa premessa fondamentale, $\mathrm{A}$. Danto ha avanzato la tesi secondo cui "la forma delle spiegazioni storiche è narrativa", spostando così il focus dalla struttura logica della spiegazione al linguaggio, al fine di individuare al suo interno le strutture proposizionali che ischiudono le interpretazioni della storia.

Per approfondimenti: DANTO, Arthur Coleman, «Proposizioni narrative», in ID., Filosofia analitica della storia, Bologna, Il Mulino, 1971, pp. 195-247.

${ }^{3}$ L.O. Mink, in una serie di articoli pubblicati tra gli anni Sessanta e Ottanta, ha sostenuto che la struttura narrativa "è un contributo al sapere e non semplicemente l'artificio letterario per la presentazione di una serie di descrizioni di fatti”. Nella sua prospettiva, però, la struttura narrativa si basa sull'interconnessione delle singole proposizioni nel testo; quindi è solo il testo a offrirci quella visione generale della rappresentazione storica.

${ }^{4}$ FOUCAULT, Michel, Le parole e le cose, Milano, BUR, 1999; ID., L'archeologia del sapere. Una metodologia per la storia della cultura, Milano, BUR, Milano, 2009; RICOEUR, Paul, Il conflitto delle interpretazioni, Milano, Jaca Book, 1995; ID., Tempo e racconto, vol. I, Milano, Jaca Book, 2008; ID., Ricordare, dimenticare, perdonare, Bologna, Il Mulino, 2012; VEYNE, Paul, Comment on écrit l'histoire, Paris, Seuil, 1971, [trad. it., Come si scrive la storia, Roma-Bari, Laterza, 1973].
} 
Estremizzando la posizione del modello retorico-narrativo, si giunge alla concezione espressa da Hayden White, il filosofo maggiormente rappresentativo di tale tradizione nel contesto anglo-americano, il quale, abbandonando definitivamente l'impostazione positivista, ha individuato nel sistema dei tropi una griglia capace di tradurre il passato in un racconto storico, concepito sotto forma di romanzo, di commedia, di tragedia, di satira o di altri generi letterari. Solo il livello della cronaca può essere collegato al concetto di verità; ma, nella sua totalità, la narrazione è una ricostruzione dello storico, satura della sua visione della realtà, dunque né vera né falsa, regno dell'immaginazione ${ }^{6}$.

Concentrando gli studi esclusivamente sugli aspetti formali della scrittura storiografica, H. White ha perso così di vista la specificità della scienza storica, ossia l'indagine, attraverso accurate problematizzazioni dell'uomo e delle società. E insistendo altresì sulla forma dei testi storiografici piuttosto che sul loro contenuto, ha contribuito a far sì che la storia e la storiografia divenissero due prodotti eminentemente soggettivi, identici a qualsiasi opera letteraria.

Il dibattito scaturito dopo la pubblicazione di Metahistory è stato molto acceso e, per certi aspetti, è ancora aperto. Si sono susseguite posizioni contrapposte, ma non sono mancati i tentativi di mediazione tra l'aspra critica alla storiografia inseritasi nel solco delle teorie di $\mathrm{H}$. White e le nuove possibilità analitiche e metodologiche proposte dal linguistic turn ${ }^{7}$.

\footnotetext{
${ }^{6}$ L'opera di H. White si inserisce nel più ampio filone della svolta linguistica che ha interessato il pensiero filosofico occidentale dalla fine del XIX secolo, raggiungendo il suo apice proprio negli anni settanta, quando è avvenuto il definitivo passaggio dal soggetto al linguaggio quale fulcro della teorizzazione filosofica nell'indagine della realtà. Si veda a questo proposito: WHITE, Hayden, «The Structure of Historical Narrative», in Clio. Journal of Literature, History, and Philosophy of History, 1-3/1972, pp. 5-20; ID., Metahistory. The Historical Imagination in Nineteenth-Century Europe, Baltimore, The Johns Hopkins University Press, 1973; ID., Tropics of Discourse: Essays in Cultural Criticism, Baltimore, The Johns Hopkins University Press, 1985; ID., Figural Realism. Studies in the Mimesis Effect, Baltimore, The Johns Hopkins University Press, 1998, pp. 176-182; ID., Storia e narrazione, Ravenna, Longo Editore, 1999; ID., «On The Public Role of History», in History and Theory, 44, 2005, pp. 311-347.

TORTAROLO, Edoardo (a cura di), Forme di storia. Hayden White. Dalla realtà alla narrazione, Roma, Carocci, 2006.

${ }^{7}$ Per ciò che concerne la corrente favorevole alla tesi di $\mathrm{H}$. White si deve ricordare il saggio di Hans Kellner: KELLNER, Hans, «A Bedrock of Order: Hayden White's Linguistic Humanism», in History and Theory. Metahistory: Six Critiques, 19, 1980, pp. 1-29. Seppur con le dovute critiche, sullo stesso versante si colloca Philip Pomper, il quale, nello stesso numero di History and Theory, definisce il progetto elaborato da H. White il miglior sistema tipologico per decodificare i prodotti culturali complessi. I detrattori, al contrario, hanno confutato le tesi di H. White, contestando soprattutto la scomparsa delle categorie di scientificità della storia. Tra questi è doveroso citare Eugene Golob, Nancy Streuver e John Nelson. Si colloca su queste posizioni, infine, Maurice Mandelbaum, che critica non solo l'eccessivo formalismo e l'utilizzo di strutture narrative a priori, ma soprattutto la sovrapposizione del pensiero filosofico a quello storiografico.
} 
La critica crescente al relativismo postmoderno e al contempo al logocentrismo, che ha posto l'accento sull'etica e sulla responsabilità dello storico, hanno contribuito, di fatto, a un certo revival of narrative, ma con nuovi strumenti e prospettive di analisi ${ }^{8}$. Sulla strada della cosiddetta concezione retorica del racconto storico, si sono avventurati, nel corso degli anni, pensatori come Stephen Banne e Ann Rigney, che hanno concentrato la loro attenzione sul livello persuasivo del racconto, mentre altri studiosi, da Susanna Gearhert, Lionel Gossman ad Arthur Mitzman, hanno disvelato altre griglie ipotetiche relative alla struttura, fino a giungere a categorie psicoanalitiche, oggi per lo più abbandonate.

La concezione più dettagliata della metodologia narratologica risale agli anni ottanta, ad opera di Franklin R. Ankersmit, secondo il quale la categoria di verità può essere applicata solo a singoli fatti isolati, mentre ravvisa nella creazione mentale dello storico la griglia strutturale del racconto9. In esso sono presenti "sostanze narrative", ossia semplici constatazioni individuali, per rappresentare le quali si riflette un particolare punto di vista che ne orienta la strutturazione. La metafora organizzatrice del racconto storico, la sua impalcatura generale, non risiede dunque nel campo della retorica, bensì nell'idea concettuale intorno alla quale ruota il racconto stesso. Egli nega, in definitiva, l'esistenza di regole di "traslazione" utilizzate dallo storico per tradurre il passato, siano esse i tropi retorici di H. White o le teorie del processo storico. In tal senso, il racconto diviene una produzione, una costruzione prevalentemente soggettiva.

Il motivo della narrazione è divenuto dunque centrale nell'ambito della filosofia della storia e non solo all'interno del pensiero postmodernista: lo ritroviamo nel modello empirico-teorico, seguito da molti pensatori ascrivibili alla cosiddetta filosofia analitica, in quello retorico-narrativo, in cui emerge in particolar modo la figura di $\mathrm{H}$. White, in quello marxista, la cui epistemologia assegna un ruolo attivo al soggetto nel processo di conoscenza ${ }^{10}$.

${ }^{8}$ Significativa al riguardo l'affermazione di J. Topolski: «È un paradosso che la critica postmoderna del logocentrismo e al contempo la critica crescente al relativismo postmoderno [...] contribuiscano entrambe allo studio dei meccanismi (della produzione) del racconto». Cfr. TOPOLSKI, Jerzy, Narrare la storia. Nuovi principi di metodologia storica, Milano, Bruno Mondadori, 1997, p. 15.

9 ANKERSMITH, Franklin, Narrative Logic. A Semantic Analysis of the Historian's Language, Boston-London-The Hague, Martinus Nijhoff Publishers, 1983; ID., «Reply to Prof. Zagorin», in History and Theory, 3/1990, pp. 275-296.

${ }^{10}$ Il narrativismo può essere considerato la declinazione del metodo linguistico della filosofia del Novecento nel dominio della storia. Esso riflette, da un lato, il metodo analitico, che comprende autori che si rifanno al realismo, con una pluralità di varianti, fino a giungere a schemi di relativismo, dall'altro, accoglie le tendenze della teoria critica, dello strutturalismo e del decostruzionismo. Arthur Danto e Frank Ankersmith hanno espresso nel modo più chiaro proprio 
In virtù delle accese dispute metodologiche, che hanno visto teorici in polemica sia con l'esasperata tendenza a trasformare la storia in una scienza arida, sia con l'azzeramento del lavoro dello storico, proposto dalle frange estreme del narrativismo, si sono difese, su vari fronti intermedi, le ragioni del narrare come mezzo storiografico irrinunciabile. Collochiamo proprio all'interno di questa posizione mediana lo storico Jerzy Topolski, espressione di una corrente minoritaria di scuola marxista, che ha tuttavia elaborato una tesi tra le più organiche ${ }^{11}$.

In definitiva, se nel filone dei narrativisti si è sottolineato in particolare il soggettivismo del ricercatore e lo scetticismo conoscitivo, su altri versanti si è tentato di coniugare la fede in una pur limitata e condizionata oggettività con le istanze del raccontare i fatti storici.

\section{Incroci metodologici}

I tratti fondamentali della linea espressa da J. Topolski risiedono proprio nel riconoscere che lo storico "costruisce" un'immagine del passato, senza abbandonare il rigore richiesto dall'empirismo logico. Nella sua veste esteriore il racconto si uniforma alle opere letterarie, ma resta tuttavia un prodotto dell'attività razionale, in particolare nella ricerca e analisi delle fonti, che continuano a costituire la materia prima su cui il racconto stesso si fonda. E alla nozione di fonte, che permette l'accesso al passato, è connesso il problema della spiegazione storica, perché in essa non si trovano mai le spiegazioni che si potrebbero riutilizzare nel racconto storico. Le strutture che guidano la narrazione non sono indipendenti dallo storico che le analizza: il controllo filologico e le proiezione nel passato dei problemi del presente s'intrecciano, condizionandosi reciprocamente, in tutti i momenti del lavoro storiografico.

il primo orientamento, mentre Hayden White, Alain Megill e Dominick LaCapra sono i massimi interpreti del secondo. Le teorie di Ankersmith sulla rappresentazione, inscrivibili nel cosiddetto modello costruttivista, poiché si tende alla "costruzione dell'immagine del passato", hanno dato nuovo vigore al dibattito sull'obiettività e sulla frammentazione disciplinare, dibattito a cui hanno partecipato importanti storici, da Carlo Ginzburg, Natalie Zemon Davis, a Emmanuel Le Roy Ladurie.

${ }^{11}$ Per approfondimenti, oltre alle opere già segnalate: TOPOLSKI, Jerzy, Metodologia della ricerca storica, Bologna, Il Mulino, 1975; ID., Microstoria e macrostoria, (problemi metodologici), Università di Perugia, Dipartimento di Scienze Storiche, Lezione 1, 1984-85; ID., «The Concept of Theory in Historical Research. Theory Versus Myth», in Storia della Storiografia, 13, 1988, pp. 67-79; ID., Problemi metodologici dell'uso delle fonti letterarie nello studio della storia, in CATALUCCIO, Francesco (a cura di), Testi letterari e conoscenza storica. La letteratura come fonte, Milano, Bruno Mondadori, 1986; ID., "The Role of Logic and Aesthetics in Constructing Narrative Wholes in Historiography», in History and Theory, 38, 1999, pp. 198-210. 
Il nucleo del modello metodologico proposto da J. Topolski, che riguarda l'analisi della struttura e della produzione del racconto storico, consiste nella distinzione nello stesso testo di tre livelli, che costituiscono una realtà narrativa, soggettiva e oggettiva allo stesso tempo, in cui il quadro fondamentale è generato sulla freccia del tempo in un dato spazio. Al livello persuasivo o retorico, segue quello informativo, logico e grammaticale, a cui si aggiunge, infine, quello teorico e ideologico, che possiamo considerare lo stadio più profondo, il motore intellettuale che definisce il contenuto dei precedenti. Se i primi due gradi trasmettono le informazioni relative al passato, il terzo comprende i meccanismi teorici che ne determinano il contenuto e la struttura.

I lavori ascrivibili al quadro della corrente narratologica della filosofia postmodernista della storia si sono indirizzati verso l'analisi del discorso, della narrazione, del linguaggio delle scienze umane. In questa prospettiva, lo storico costruisce il suo racconto con la consapevolezza che il suo narrare non è un procedimento informativo, ma meramente narrativo, autenticamente retorico. È una ricerca senza verità in assoluto, nelle posizioni più radicali, o disposta a concedere un margine di realismo soltanto in termini di semplici fatti individuali di microstoria, nelle posizioni meno radicali, mentre le constatazioni generali rimangono di fatto non "reali".

Il passaggio al terzo livello è stato condizionato da significativi cambiamenti intervenuti nel campo storiografico, che hanno costituito uno stimolante incentivo all'analisi teorico-esplicativa. Un terreno significativo sul quale misurare la distanza con il progetto filosofico postmodernista sulla storia è costituito proprio dal valore della fonte, in quanto essa esprime un'esigenza ineliminabile della pratica storica e una componente importante per scrivere la storia, a fianco della inventio, della descrizione e dell'argomentazione.

I problemi connessi alla spiegazione e alla rappresentazione storica sono stati a lungo oggetto di dibattito, a volte istaurando un dialogo virtuoso sia con la tradizione sia con il narrativismo storico. Per lo scopo di questo scritto, è rilevante soffermarsi sull'incontro e sullo scontro di alcuni storici del calibro di Carlo Ginzburg, Natalie Zemon Davis e Le Roy Ladurie con gli esponenti più importanti del narrative turn.

Prendiamo in considerazione tre libri di storia che hanno influito notevolmente sulla svolta storiografica compiuta nell'ultimo scorcio del Novecento ${ }^{12}$. La microstoria

${ }^{12}$ LE ROY LADURIE, Emmanuel, Storia di un paese: Montaillou. Un villaggio occitanico durante l'inquisizione (1294-1324), Milano, Rizzoli, 1977; GINZBURG, Paul, Il formaggio e $i$ vermi. Il cosmo di un mugnaio del '5oo, Torino, Einaudi, 1976; ZEMON DAVIS, Natalie, Il ritorno di Martin Guerre. Un caso di doppia identità nella Francia del Cinquecento, Torino, Einaudi, 1984. 
di Carlo Ginzburg, la storia regionale di Emmanuel Le Roy Ladurie, la storia culturale di Natalie Zemon Davis hanno molti punti di contatto. Senza soffermarsi sui dettagli, basti ricordare la comune focalizzazione su realtà regionali e locali, su individui o gruppi marginali, l'interesse per le questioni periferiche, la ricerca del fatto imprevedibile e irripetibile, la scala ridotta della rappresentazione e la rivendicazione del ruolo dell'anomalia a scapito del riferimento a processi seriali. Queste contiguità non devono oscurare le differenze esistenti tra microstoria, storia regionale e nuova storia culturale. Nel caso della microstoria, ad esempio, la focalizzazione sulle esperienze di gruppi marginali e individui perseguitati è ispirata da una critica politica e ideologica del potere, e delle sue strategie repressive, mentre le indagini degli storici della cultura, come Zemon Davis o Stedman Jones, sono animate da un interesse per il discorso di genere e per la costruzione sociale dell'identità femminile. Così come non si devono offuscare le critiche rivolte alla svolta linguistica da parte di alcuni storici particolarmente impegnati sul fronte della discussione teorica.

Il ricorso alla forma del racconto diventa, per Carlo Ginzburg, uno strumento straordinariamente duttile di scavo gnoseologico: non si tratta di una forma apportata al dato documentario, o alle proposizioni descrittive che possono essere tratte da esso, così da organizzarlo secondo principi linguistici estranei al documento stesso. Tra l'uno e l'altro, tra documento e racconto, vi è un rapporto più sottile e intricato. La narrazione incorpora ed esplicita gli ostacoli, le tecniche, le implicazioni insite nel processo della ricerca, lascia trasparire la funzione di «filtri e intermediari deformanti» che a volte i documenti hanno. Essa, in breve, non coincide sic et simpliciter con l'intramazione retorica o non è solo riducibile ad aspetti stilistici, ma riflette il processo della ricerca, denunciando il valore ideologico, a volte occultante, della documentazione.

Anche il testo di Le Roy Ladurie non si limita a ricostruire una vicenda individuale: la racconta, utilizzando una forma che riflette le evoluzioni metodologiche e concettuali implicate nella nouvelle histoire, una dizione usata intorno agli anni settanta nel circolo delle Annales, per indicare nell'insieme i nuovi approcci storiografici. Evidentemente il racconto non è più sintomo di una visione teleologica centrata sulle gesta di personaggi di primo piano, ha perso la connotazione etnocentrica, ruotante attorno ai concetti di nazione, borghesia, missione civilizzatrice, quindi non è più allineato con i principi unificatori della histoire événementielle.

Anzitutto il racconto deve rendere le diverse focalizzazioni tematiche, gli spostamenti d'interesse e pertinenza, in secondo luogo deve restituire i mutamenti metodologici e le variazioni della ricerca. È quest'ultimo un dato da non sottovalutare, 
perché conduce a una riformulazione, non all'abbandono, del carattere essenzialmente unitario o unificante del racconto. Gli ostacoli frapposti alla ricerca, nelle nuove esperienze storiografiche entrano a far parte del racconto stesso: «le ipotesi, i dubbi, le incertezze diventano parti della narrazione», «le distorsioni e le lacune dei documenti», le stesse «implicazioni gnoseologiche» sono trasformate in elemento narrativo ${ }^{13}$.

Carlo Ginzburg individua, tuttavia, nelle teorie narrativistiche, in particolare nelle tesi di H. White, il pericolo di una polverizzazione delle prove e, quindi, dell'attendibilità delle testimonianze, che minaccia la possibilità stessa di una ricostruzione veritiera del passato. La prova deve rimanere essenziale nella narrazione storiografica, soprattutto per distinguere quest'ultima dalla letteratura di finzione. Il limite della fonte può essere considerato interno alla situazione della ricerca stessa, ma questo non autorizza lo storico ad attribuire al passato il significato che vuole, perché la ricerca implica sempre un equilibrio tra le diverse modalità del discorso (denotativa, connotativa, performativa), le propensioni ideologiche e le forme dell'argomentazione.

\section{La lezione di Jerzy Topolski}

All'interno del nuovo contesto metodologico sulla spiegazione storica, la lezione di J. Topolski andrebbe riletta e valorizzata, perché da qui si può riaprire il dibattito storiografico, nella prospettiva del rapporto tra la linguistica, il narrativismo e la storia, che ha avuto troppo marginali occasioni di sviluppo ${ }^{14}$.

L'autore propone un'analisi equilibrata di tutti i livelli di cui si compone il racconto storico, in una combinazione senza drastiche fratture con la ricostruzione fattografica del passato, che rimane l'elemento indispensabile nello studio della pratica storiografica, nonostante siano largamente privilegiati l'aspetto retorico e le forze latenti che ne muovono i meccanismi.

${ }^{13}$ Cfr. GINZBURG, Carlo, Il filo e le tracce. Vero, falso, finto, Milano, Feltrinelli, 2006, pp. 256262.

${ }^{14}$ Tra gli interventi significativi si segnalano: STONE, Lawrence, «The Revival of Narrative: Reflections on a New Old History», in Past and Present, 85, 1979, pp. 3-24; RORTY, Richard, La svolta linguistica, Milano, Garzanti, 1994; GHIGNOLI, Antonella, «La spiegazione storica: dibattiti recenti», in Bullettino dell'Istituto storico italiano per il Medio Evo, 111, 2009, pp. 464481; SHIELDS, David, Fame di realtà. Un manifesto, Roma, Fazi, 2010; CORTELLA, Lucio, Dal soggetto al linguaggio. Un percorso nella filosofia contemporanea, Venezia, Libreria Editrice Cafoscarina, 2012; SOCRATE, Francesca, SORBA, Carlotta (a cura di), Contemporanea : Tra linguistica e storia: incroci metodologici e percorsi di ricerca, 2, 2013; STEINTHAL, Heymann, BONDİ, Davide (a cura di), Ermeneutica e psicologia del linguaggio. Scritti, Milano, Bompiani, 2013; BONDİ, Davide, La teoria della storia, Milano, Unicopli, 2013; ID., Filosofia e storiografia nel dibattito anglo-americano sulla svolta linguistica, Firenze, Firenze University Press, 2014. 
I tratti più evidenti della linea espressa dallo storico polacco sono il riconoscimento che nella sua "veste esteriore" il racconto di uniforma alle opere letterarie, ma che esso resta, nello stesso tempo, un prodotto dell'attività razionale dello storico, senza escludere l'influenza delle componenti emozionali.

Lo storico si trova di fronte alla produzione del racconto quando dal piano pragmatico-metodologico, strettamente interconnesso alla procedura di ricerca, passa all'esposizione dei risultati delle indagini compiute, che si traduce in proposizioni storiche fra loro collegate in modo coerente su un dato frammento di realtà. Ciò significa che si devono mettere insieme i tasselli per comporre l'unità, nella quale le informazioni, le conoscenze e i dati siano fusi in una creazione strutturata e cristallina.

La narrazione rappresenta il problema centrale della metodologia apragmatica delle scienze e, in ogni caso, della metodologia apragmatica della storia, che considera il racconto il medium di comunicazione. Spetta allo storico, dunque, argomentare il messaggio e farlo comprendere ai destinatari, utilizzando un linguaggio con il quale siano veicolati i fenomeni del passato, gli scenari, gli strumenti di indagine utilizzati, le tematiche, i problemi e i soggetti. E fanno parte del linguaggio la grammatica e le regole semantiche, a cui si attengono i vocaboli che alle proposizioni attribuiscono determinati significati. Scegliere di inserire nel testo una terminologia specifica non solo determina una precisa presa di posizione storiografica e ideologica, ma implica anche una differente sollecitazione nei confronti del destinatario. Optare per un tono apologetico, consenziente, oppure di distacco e di disapprovazione rende evidente l'ottica interpretativa dell'autore, tenendo per fermo che lo storico deve possedere quell'obiettività e onestà intellettuale necessarie nella ricostruzione dei fatti, a partire dalla prova fornita dalle fonti.

La storia rientra nell'ambito delle scienze che si servono dei linguaggi naturali e la narrazione è stata e rimane il modello esclusivo per la comunicazione dei risultati della ricerca, largamente praticato anche all'interno della comunità scientifica. Sono frequenti tuttavia nella narrazione concetti ordinatori di vario genere che adempiono la funzione di organizzazione della conoscenza. E proprio perché allo storico preme in primis trasmettere e far comprendere il reale decorso degli avvenimenti, è opportuno far riferimento a definizioni il più possibile incisive, precisate nell'ambito di una determinata scienza che se ne occupa professionalmente e alla loro spiegazione dettagliata quando assumono differenti curvature.

Per la costruzione della narrazione storica, sfruttando la costante prospettiva temporale, sono necessari numerosi strumenti, che sono componenti delle conoscenze extra-fonti oppure funzione delle medesime. A tali mezzi appartengono: 
l’immaginazione storica, il linguaggio, i concetti di classificazione, la logica, la retorica, ma anche le conoscenze derivate da altre scienze ausiliarie, che sono inseriti nella cassetta degli attrezzi dello storico. E per collegare i vari elementi è necessaria la capacità di movimentare i saperi accumulati, nell'intento di generare un'equilibrata visione d'insieme, che non esclude operazioni di generalizzazione. Esse sono, secondo J. Tolopski, stricto sensu oppure "quasi statistiche", ed entrano profondamente nei meccanismi di spiegazione storica. Dai dati raccolti in modo oggettivo, è possibile passare alle formule del "di regola", del "molto spesso", che nella pratica finiscono con l'essere equivalenti al dato statistico.

Il testo che ne deriva segue dunque un percorso informativo, logico, narrativo e di idealizzazione, che si sviluppa in proposizioni storiche correlate a precise coordinate spazio-temporali, sia per singoli eventi o brevi periodi, sia per fenomeni di lunga durata. La catena narrativa risulta così composta da una sequenza di asserzioni la cui organizzazione è varia, ma subordinata sempre e comunque al rigore metodologico.

Non esiste tuttavia per J. Tolopski solo la successione di proposizioni storiche, che descrivono fatti collettivi. Esse sono calate nell'oceano delle generalizzazioni, delle nozioni teoriche, dunque nella concettualizzazione messa a punto dallo storico. Siamo di fronte a un fenomeno che è connesso con la teoria del racconto storico e più precisamente a complessi intrecci tra frasi e immagini storiche. Così una frase storica, cioè l'informazione su un determinato fatto, appartiene contemporaneamente a immagini sempre più estese a mano a mano che il racconto si sviluppa. E questo complessità, nel racconto coerente, abbraccia tutto il contenuto in modo articolato.

Per una visione di più ampio respiro, è particolarmente significativo ricorrere al catalogo tipologico proposto da J. Topolski, che distingue numerose "coppie": racconto descrittivo e giustificativo, fattuale e teorico, monografico e sintetico, solo per citare i binomi più significativi ${ }^{15}$.

La prima coppia sottolinea una vera e propria diramazione nella trasmissione del sapere storico, poiché propone, da una parte, un prodotto completo e definito dai risultati della ricerca assunti come definitivi, dall'altra, vi è la volontà di presentare i modi e gli strumenti con i quali sono stati conseguiti i risultati offerti al lettore, evidenziando quelle ipotesi, quelle piste che nel corso della ricerca sono state abbandonate, perché prive di un valido riscontro. Una sorta di narrazione aperta a più proposte di sintesi, più disponibile a successive integrazioni, a riprese e recuperi, anche dopo la conclusione dello studio. Chi adotta questa modalità può essere considerato

\footnotetext{
${ }^{15}$ Per una disamina dettagliata, ved. TOPOLSKI, Jerzy, Narrare la storia, cit., 1997, p. 21.
} 
uno "storico-enzima", poiché contribuisce a «far transitare il passato nel presente, rendendolo assimilabile, digeribile, utilizzabile» ${ }^{16}$.

Il racconto teorico costituisce, rispetto a quello fattuale, ossia legato alla descrizione dei fatti storici nella loro realtà oggettiva, il motore di tutta l'operazione storiografica, la base su cui poggia l'intento di ricostruzione. E il linguaggio utilizzato in questo contesto risente ovviamente delle rappresentazioni mentali, del sapere pregresso, del sistema dei valori cui fa riferimento, dei modelli ordinatori e dei paradigmi che organizzano l'attività intellettuale dello storico. Sono tali, ad esempio, i concetti di continuità e di cambiamento, il principio di coerenza e di relazione all'interno del discorso, oppure altre categorie ordinatrici. Esiste, tuttavia, anche una dimensione teorica vera e propria interna o desunta da altre discipline diverse dalla storia; è il caso dell'analisi quantitativa oppure di teorie economiche o sociologiche, da cui si possono prendere in prestito indicatori e modelli di spiegazione.

L'ultima coppia, relativa al racconto monografico e sintetico, segna nel profondo la produzione scientifica accademica; si tratta, in questo caso, di coniugare i lavori di ricerca su temi specialistici con le messe a punto di tematiche, di processi, di situazioni di più ampio respiro, che giungono sino alla storia globale.

Microstoria e macrostoria non si distinguono per il metodo, ma solo per l'estensione del loro oggetto di ricerca. Certo le nozioni utilizzate dallo storico per la narrazione sintetica, tipica della storia non locale, sono più generali di quelle adottate in lavori analitici, ma è una differenza di grado e non di sostanza.

\section{Riflessioni conclusive}

Il rapporto tra lavoro scientifico e la narrazione è, dunque, un'operazione complessa, che si traduce in una necessaria mediazione tra il valore logicoargomentativo e l'immaginazione che influenza il percorso di ricostruzione del passato. Esso avviene attraverso l'utilizzo del linguaggio, che non è un mezzo neutrale attraverso il quale esprimere gli esiti del processo cognitivo, fermo restando, in ogni caso, che la specificità del lavoro dello storico risiede sempre e comunque nel costante e antiletterario richiamo alle fonti.

La produzione storica, come ha acutamente rilevato J. Topolski, si caratterizza proprio per "la volontà dello storico di proporre una spiegazione più estesa di fatti ai

${ }^{16}$ Cfr. DE LUNA, Giovanni, La passione e la ragione. Il mestiere dello storico contemporaneo, Milano, Bruno Mondadori, 2004, p. 63. 
quali egli attribuisce un'importanza maggiore" 17 . È chiaro che la spiegazione può calarsi nel racconto storico senza interromperne la narrazione; tuttavia, laddove si manifesta esplicitamente, essa può avvenire narrativamente e in modo dimostrativo, oppure può essere deduttiva. Nel proprio processo esplicativo, lo storico può ricorrere al procedimento della giustificazione o dell'argomentazione, ossia a una spiegazione logicamente giustificata oppure a un certo grado di giustificazione, sempre revocabile e parzialmente dipendente dalla logica.

$\mathrm{Ne}$ deriva una nuova filosofia dell'argomentazione che pone l'accento sulla formazione del sapere, rifiutando l'esistenza di una verità unica e privilegiata, in favore di un racconto storico sia come costruzione sia come processo culturale.

Per J. Topolski la vera spiegazione storica consiste nella ricerca della "rete" di cause individuali che hanno provocato l'esistenza di un fenomeno, e non nell'integrazione di quel fenomeno in una "struttura" precostituita. La spiegazione delle azioni umane, unitamente alla spiegazione dei fatti e dei processi, realizzata attraverso generalizzazioni di diverso livello, costituiscono un cerchio all'interno del quale porre il racconto.

Sul versante opposto, affermare che il fatto e la realtà non posseggano altro che un'esistenza linguistica, che siano cioè costruiti soltanto nel discorso e che esistano soltanto in esso, significa per l'autore "derealizzare" la realtà in sé, dunque la materia stessa della storia, e aprire la strada alla illimitata capacità del linguaggio di manipolare indefinitamente gli eventi del passato, di crearli come di negarli: una posizione che può diventare foriera di pericoli, per il relativismo che può innescare fuorvianti revisioni.

Constatato che nel corso degli anni è stato privilegiato lo strato fattografico, J. Topolski ci invita a scendere nelle profondità per far emergere ciò che è latente, ossia il terzo strato, quello che determina la selezione delle informazioni, la loro gerarchizzazione e la costruzione formale del racconto.

Il mondo narrativo del racconto è concepito, in definitiva, come una totalità ricostruita, perché la storia è scelta, deformata dalle selezioni preliminari e successive operate dallo storico. Il filo garantito dalle fonti, che legano la narrazione al passato, è troppo fragile perché si possa accostare il racconto alla realtà: esso non trasmette l'immagine, la mappa o il modello del passato, ma soltanto informazioni sparse. E dunque la metafora sartoriale del "filo che visualizza un contatto" con la realtà passata, che si avvicina o tenta di avvicinarsi alla verità storica, è la sintesi creativa che unisce la cornice teorica, il rigore logico deduttivo all'invenzione e alle risorse mentali, che scaturiscono dal sapere pregresso e dal sistema di valori in cui è immerso l'autore.

${ }^{17}$ Cfr. TOPOLSKI, Jerzy, Narrare la storia, cit., 1997, p. 164. 


\section{* L'autore}

Patrizia Fazzi, docente a contratto di Storia contemporanea presso il Dipartimento di Studi Umanistici dell'Università di Torino, insegnante di materie letterarie in un liceo torinese, si occupa di didattica della storia e di sociologia delle migrazioni. Tra le pubblicazioni si segnalano: Migrazioni e trasformazione sociale in Italia. Dall'età moderna a oggi, Milano, FrancoAngeli, 2008; in collaborazione con il Dipartimento di Scienze sociali dell'Università di Torino: $I l$ Movimento degli obiettori alle spese miliari in Italia, in ANFOSSI, Anna, OOMMEN, Tharailath Koshy (a cura di), Azioni politiche fuori dei partiti, Milano, FrancoAngeli, 1997, pp. 85-105; tra i saggi si segnala: «La guerra in Cecenia: un nazionalismo mai sopito», in Giano, 22, 1996, pp. 1931. In ambito didattico: «Problemi, metodi e didattica della storia moderna e contemporanea», in Storia e Futuro, 35, 2/2014, pp. 1-24.

URL: < http://www.studistorici.com/progett/autori/\# Fazzi >

\section{Per citare questo articolo:}

FAZZI, Patrizia, «Narrare la storia: la lezione di Jerzy Topolski», Diacronie. Studi di Storia Contemporanea : Costruire. Rappresentazioni, relazioni, comunità, 29/06/2015,

URL:< http://www.studistorici.com/2015/06/29/fazzi_numero_22/ >

Diacronie Studi di Storia Contemporanea 3 www.diacronie.it

Risorsa digitale indipendente a carattere storiografico. Uscita trimestrale. redazione.diacronie@hotmail.it

Comitato di redazione: Jacopo Bassi - Luca Bufarale - Elisa Grandi - Deborah Paci - Fausto Pietrancosta - Matteo Tomasoni - Luca Zuccolo

Diritti: gli articoli di Diacronie. Studi di Storia Contemporanea sono pubblicati sotto licenza Creative Commons 2.5. Possono essere riprodotti a patto di non modificarne i contenuti e di non usarli per fini commerciali. La citazione di estratti è comunque sempre autorizzata, nei limiti previsti dalla legge. 